PROCEEDINGS OF THE

AMERICAN MATHEMATICAL SOCIETY

Volume 135, Number 4, April 2007, Pages 1175-1180

S 0002-9939(06)08586-8

Article electronically published on October 18, 2006

\title{
COMPACT PERTURBATIONS OF ISOMETRIES
}

\author{
IOANA SERBAN AND FLAVIUS TURCU
}

(Communicated by Joseph A. Ball)

\begin{abstract}
We give some characterizations of isometries (contractions) which are perturbations with compact operators of a given arbitrary isometry, in terms of certain natural factorizations. As a consequence we obtain general parametric representations of these perturbations.
\end{abstract}

\section{INTRODUCTION}

Let $L, H$ be separable, infinite-dimensional Hilbert spaces and $B(L, H)$ the Banach space of the bounded linear operators from $L$ to $H$. For a closed subspace $H_{0}$ of $H$, we denote by $\mathbf{1}_{H_{0}}$ the identity on $H_{0}$ and by $\iota_{H_{0}}$ the embedding of $H_{0}$ into $H$.

In recent years several works have dealt with the study of the perturbations $V_{2}=V_{1}+X$ of an isometry $V_{1} \in B(L, H)$, where $X$ ranges over particular subsets of the Banach space $K(L, H)$ of the compact operators in $B(L, H)$, such as rank one operators ([Nak86], Nak93]) or finite rank operators (BT97, [BT00]). A first question in this study is the description of all such perturbations $V_{2}$ that remain isometric or, at least, contractive. Such descriptions are given in Nak86, Nak93. for $X$ of rank one; namely, $V_{2}=V_{1}+X$ is an isometry (respectively contraction) if and only if

$$
X=(\alpha-1) h \otimes V_{1}^{*} h
$$

for some unitary vector $h \in H$ and some complex scalar $\alpha$ such that $|\alpha|=1$ (respectively $|\alpha| \leq 1$ ).

By a direct computation, this is in fact equivalent to a factorization of the type

$$
V_{2}=Y V_{1}
$$

for some unitary (respectively contractive) $Y \in B(H)$ such that $Y-\mathbf{1}_{H}$ is a rank one operator (namely $Y=\mathbf{1}_{H}+(\alpha-1) h \otimes h$ ).

The aim of this paper is to extend this characterization in the factored form (1.2) to the case when $X$ ranges over the finite rank operators and over the whole $K(L, H)$. As a consequence, the spectral description of the factor $Y$ yields parametric characterizations of the type (1.1).

Let us see first how a factorization of type (1.2) can be obtained for a contractive finite rank perturbation of an isometry.

Received by the editors May 25, 2005 and, in revised form, November 17, 2005.

2000 Mathematics Subject Classification. Primary 47A55; Secondary 47A20, 47B07.

(C)2006 American Mathematical Society 
Proposition 1.1. Let $V_{1}$ be an isometry and $V_{2}$ a contraction (respectively an isometry) in $B(L, H)$. Then $V_{2}-V_{1}$ is a finite rank operator if and only if there is a contraction (respectively a unitary) $Y$ in $B(H)$ such that $V_{2}=Y V_{1}$ and $Y-\mathbf{1}_{H}$ is a finite rank operator.

Proof. Suppose $V_{2}$ is a contraction, put $X=V_{2}-V_{1}$ and let $\mathcal{X}=\operatorname{Im} X$ (which has finite dimension). One can observe that $\mathcal{X} \cap \operatorname{Ker} V_{1}^{*}=\{0\}$. Indeed, if $x=X y$ is a vector in $\mathcal{X} \cap \operatorname{Ker} V_{1}^{*}$, then

$$
\|x\|^{2}=\left\langle V_{1}^{*} X y, y\right\rangle+\left\langle y, V_{1}^{*} X y\right\rangle+\|X y\|^{2}=\left\|\left(V_{1}+X\right) y\right\|^{2}-\left\|V_{1} y\right\|^{2} \leq 0,
$$

so necessarily $x=0$.

Let $P$ be the orthogonal projection onto $\mathcal{X}$. Since $X^{*}\left(\mathbf{1}_{H}-P\right)=\left(\mathbf{1}_{H}-P\right) X=0$, the inequality

$$
\left(V_{1}+X\right)^{*}\left(V_{1}+X\right)^{*} \leq \mathbf{1}_{H}=V_{1}^{*} V_{1}
$$

is equivalent to

$$
\left(V_{1}^{*}+X^{*}\right) P\left(V_{1}+X\right)+V_{1}^{*}\left(\mathbf{1}_{H}-P\right) V_{1} \leq V_{1}^{*} P V_{1}+V_{1}^{*}(I-P) V_{1}
$$

or

$$
\left(V_{1}^{*}+X^{*}\right) P\left(V_{1}+X\right) \leq V_{1}^{*} P V_{1} .
$$

Thus the map $Y_{0}: P V_{1} x \mapsto P\left(V_{1}+X\right) x$ is a contraction defined on $P V_{1} L$. But $P V_{1} L=\mathcal{X}$ since $\mathcal{X} \cap \operatorname{Ker} V_{1}^{*}=\{0\}$. Then $Y \in B(H)$ defined by $Y=\mathbf{1}_{H}-P+Y_{0} P$ is the required contraction.

If $V_{2}$ is supposed to be an isometry, then all the inequalities above become equalities, which implies that $Y$ is a unitary, and so the direct implication is proved. The converse is trivial.

\section{FACTORIZATIONS AND PERTURBATIONS With COMPACT OPERATORS}

Let $V_{1} \in B(L, H)$ be an isometry and $V_{2} \in B(L, H)$ a contraction. Since $V_{2}^{*} V_{2} \leq$ $V_{1}^{*} V_{1}$ there are contractions $Y \in B(H)$ such that $V_{2}=Y V_{1}$. Two such contractions necessarily coincide on $\operatorname{Im} V_{1}$, as they are all extensions of the contraction $Y_{0}$ defined on $\operatorname{Im} V_{1}$ into the closure of $\operatorname{Im} V_{2}$ by $Y_{0}: V_{1} l \mapsto V_{2} l, l \in L$. Clearly $V_{2}$ has closed range if and only if $Y_{0}$ has closed range.

In particular, if $V_{2}$ is an isometry, then $Y_{0}$ is a unitary, so one can find extensions $Y$ that are at least partial isometries. Obviously if $\operatorname{Im} V_{1}$ and $\operatorname{Im} V_{2}$ have the same codimension, then $Y_{0}$ can be extended for instance to unitaries of the form $Y=Y_{0} \oplus Y_{1}$, with $Y_{1}$ an arbitrary unitary sending $H \ominus \operatorname{Im} V_{1}$ onto $H \ominus \operatorname{Im} V_{2}$.

The question now is what else can be said about the factors $Y$ under the additional assumption that $V_{2}$ is a contractive perturbation of $V_{1}$ with a compact operator. Several remarks are immediate in this case:

First it follows that $V_{2}$ is right semi-Fredholm, so in particular $Y_{0}$ has closed range. Moreover, if $V_{2}$ is an isometry, then its range has the same codimension as $V_{1}$, so, as remarked, there are plenty of unitary extensions $Y$ of $Y_{0}$ satisfying (1.2).

Secondly, it follows from (1.2) that the operator $\iota_{\operatorname{Im}} V_{2} Y_{0}-\iota_{\operatorname{Im} V_{1}}$ is necessarily compact. The aim of this section is to show that $V_{2}-V_{1}$ is compact if and only if there is one extension $Y$ that inherits this last property of $Y_{0}$, namely $Y-\mathbf{1}_{H}$ is compact. This is obviously true for any extension $Y$ of $Y_{0}$ if $\operatorname{Im} V_{1}$ has finite codimension, so in the following we consider the nontrivial case when $\operatorname{Im} V_{1}$ has infinite codimension. 
We first consider the particular case when the perturbation $V_{2}$ is an isometry. For $i=1,2$ set $H_{i}=V_{i} L$ and denote by $P_{i}$ the orthogonal projection onto $H_{i}$. If $V_{1}-V_{2}$ is compact, then so is $P_{1}-P_{2}$, since

$$
P_{1}-P_{2}=\frac{1}{2}\left[\left(V_{1}-V_{2}\right)\left(V_{1}^{*}+V_{2}^{*}\right)+\left(V_{1}+V_{2}\right)\left(V_{1}^{*}-V_{2}^{*}\right)\right] .
$$

It is useful to investigate first the geometry of two arbitrary closed subspaces $H_{1}$ and $H_{2}$ of $H$ whose projections have compact difference. The next lemma shows that such subspaces always contain finite-codimensional parts which can be mapped one onto the other by unitaries that differ from the identity by a compact operator.

Lemma 2.1. Let $H_{1}$ and $H_{2}$ be infinite-dimensional subspaces of $H$. Let $P_{1}$ and $P_{2}$ be the orthogonal projections onto $H_{1}$ and $H_{2}$ respectively, and suppose that $P_{1}-P_{2}$ is compact. Then

1) $H_{1} \cap H_{2}^{\perp}$ and $H_{2} \cap H_{1}^{\perp}$ have finite dimensions, say $c_{1}$ and $c_{2}$.

2) There is a unitary $U_{0}$ mapping $H_{1}^{\prime}=H_{1} \ominus\left(H_{1} \cap H_{2}^{\perp}\right)$ onto $H_{2}^{\prime}=H_{2} \ominus$ $\left(H_{2} \cap H_{1}^{\perp}\right)$ such that $\iota_{H_{2}^{\prime}} U_{0}-\iota_{H_{1}^{\prime}}$ is compact.

3) If, for example, $c_{1} \leq c_{2}$, then $U_{0}$ can be extended to an isometry $V_{0}$ from $H_{1}$ into $\mathrm{H}_{2}$ satisfing

a) $\iota_{H_{2}} V_{0}-\iota_{H_{1}}$ is compact;

b) $\operatorname{dim}\left(H_{2} \ominus V_{0} H_{1}\right)=c_{2}-c_{1}$.

In particular if $c_{1}=c_{2}$, then $V_{0}$ can be chosen to be unitary.

Proof. To prove 1), observe that $\mathbf{1}_{H_{1} \cap H_{2}^{\perp}}=\left.\left(P_{1}-P_{2}\right)\right|_{H_{1} \cap H_{2}^{\perp}}$, so $\mathbf{1}_{H_{1} \cap H_{2}^{\perp}}$ is compact, and this happens if and only if $\operatorname{dim}\left(H_{1} \cap H_{2}^{\perp}\right)<\infty$. In a similar way it follows that $\operatorname{dim}\left(H_{2} \cap H_{1}^{\perp}\right)<\infty$.

To prove 2), let $X: H_{1} \rightarrow H_{2}$ be the operator angle between $H_{1}$ and $H_{2}$, i.e., the restriction of $P_{2}$ to $H_{1}$. Obviously $X^{*}$ is the reverse operator angle, i.e., the restriction of $P_{1}$ to $H_{2}$. Since $P_{2}-P_{1}$ is compact, it follows immediately that $\iota_{H_{2}} X-\iota_{H_{1}}$ is compact.

We also have Ker $X=H_{1} \cap H_{2}^{\perp}$ and Ker $X^{*}=H_{2} \cap H_{1}^{\perp}$, so the restriction $X_{0}$ of $X$ to the subspace $H_{1}^{\prime}=H_{1} \ominus$ Ker $X$ considered as an operator from this subspace into $H_{2}^{\prime}=H_{2} \ominus \operatorname{Ker} X^{*}=\overline{\operatorname{Im} X}$ is a quasi-invertible operator (i.e., injective with dense range) from $H_{1}^{\prime}$ to $H_{2}^{\prime}$ for which $\iota_{H_{2}^{\prime}} X_{0}-\iota_{H_{1}^{\prime}}$ is compact.

Now, since for $h \in H_{1}^{\prime}$,

$$
X_{0}^{*} X_{0} h-h=P_{1} P_{2} h-h=P_{1} P_{2} h-P_{1} h=P_{1}\left(P_{2}-P_{1}\right) h,
$$

we have that $X_{0}^{*} X_{0}-\mathbf{1}_{H_{1}^{\prime}}$ is compact. This implies easily that $\left|X_{0}\right|=\left(X_{0}^{*} X_{0}\right)^{1 / 2}$ is invertible in $H_{1}^{\prime}$ and that $\left|X_{0}\right|-\mathbf{1}_{H_{1}^{\prime}}$ is compact, and therefore $\left|X_{0}\right|^{-1}-\mathbf{1}_{H_{1}^{\prime}}=$ $\left(\mathbf{1}_{H_{1}^{\prime}}-\left|X_{0}\right|\right)\left|X_{0}\right|^{-1}$ is also compact.

We set $U_{0}=X_{0}\left|X_{0}\right|^{-1}$, which is a unitary from $H_{1}^{\prime}$ onto $H_{2}^{\prime}$. Then

$$
\iota_{H_{2}^{\prime}} U_{0}-\iota_{H_{1}^{\prime}}=\left(\iota_{H_{2}^{\prime}} X_{0}-\iota_{H_{1}^{\prime}}\right)\left|X_{0}\right|^{-1}+\iota_{H_{1}^{\prime}}\left(\left|X_{0}\right|^{-1}-\mathbf{1}_{H_{1}^{\prime}}\right)
$$

is compact, which proves 2). Statement 3) is an immediate consequence of 2).

Now, under the stronger assumption that $H_{1}$ and $H_{2}$ are not only ranges of projections but ranges of isometries with compact difference, then one can find unitaries differing from the identity by a compact operator which act not only between some finite-codimensional parts of $H_{1}$ and $H_{2}$, but between $H_{1}$ and $H_{2}$ themselves. 
Theorem 2.2. Let $H_{1}, H_{2}$ be infinite-dimensional closed subspaces of $H$, and let $P_{i}$ be the orthogonal projection onto $H_{i}(i=1,2)$. The following are equivalent:

1) there exist two isometries $V_{1}, V_{2}$ in $B(L, H)$ with ranges $H_{1}$ and $H_{2}$ respectively, and $V_{1}-V_{2}$ compact;

2) there is a unitary $U: H_{1} \rightarrow H_{2}$ such that $\iota_{H_{2}} U-\iota_{H_{1}}$ is compact;

3) $P_{1}-P_{2}$ is compact and $\operatorname{dim}\left(H_{1} \cap H_{2}^{\perp}\right)=\operatorname{dim}\left(H_{2} \cap H_{1}^{\perp}\right)$.

Proof. The implication 1) $\Rightarrow 2$ ) is contained in the remarks at the beginning of this section (with $U=Y_{0}$ ). For the converse pick any isometry $V_{1} \in B(L, H)$ with range $H_{1}$ (this is possible since $L$ has infinite dimension) and put $V_{2}=U V_{1}$. The implication 3) $\Rightarrow 2$ ) follows directly from Lemma 2.1 with $c_{1}=c_{2}$.

It remains to show that 1$) \Rightarrow 3$ ). Obviously $P_{1}-P_{2}$ is compact, so by Lemma 2.1 the dimensions $c_{1}=\operatorname{dim}\left(H_{1} \cap H_{2}^{\perp}\right)$ and $c_{2}=\operatorname{dim}\left(H_{2} \cap H_{1}^{\perp}\right)$ are finite. To see that they are equal, suppose for example that $c_{1} \leq c_{2}$. Then by Lemma 2.1 there is an isometry $V_{0}: H_{1} \rightarrow H_{2}$ such that $\operatorname{dim}\left(H_{2} \ominus V_{0} H_{1}\right)=c_{2}-c_{1}$ and $\iota_{H_{2}} V_{0}-\iota_{H_{1}}$ is compact.

Due to the equivalence of 1) and 2), one can also find a unitary $W$ from $H_{1}$ to $H_{2}$ such that $\iota_{H_{2}} W-\iota_{H_{1}}$ is compact. Therefore $W-V_{0}: H_{1} \rightarrow H_{2}$ is compact, and so $W W^{*}-V_{0} W^{*}=\mathbf{1}_{H_{2}}-V_{0} W^{*}$ is compact. Since the isometry $V_{0} W^{*}$ has the range of codimension $c_{2}-c_{1}<\infty$ and since the difference of the two isometries $\mathbf{1}_{H_{2}}$ and $V_{0} W^{*}$ is compact, we have

$$
0=\left(\operatorname{dim} H_{2} \ominus \mathbf{1}_{H_{2}} H_{2}\right)=\operatorname{dim}\left(H_{2} \ominus V_{0} W^{*} H_{2}\right)=c_{2}-c_{1},
$$

so $c_{1}=c_{2}$ and the theorem is proved.

The characterization 3 ) in Theorem 2.2 in terms of projections allows a nice interplay between (infinite-codimensional) subspaces and their orthogonal complements, which leads to the main result of the section.

Theorem 2.3. Let $V_{1}, V_{2}$ be arbitrary isometries in $B(L, H)$. Then $V_{1}-V_{2}$ is compact if and only if there is a unitary $U$ in $B(H)$ such that $U-\mathbf{1}_{H}$ is compact and $V_{2}=U V_{1}$.

Proof. Let $H_{i}=V_{i} L, i=1,2$. As mentioned in the beginning of the section we may suppose that $K_{1}=H \ominus H_{1}$ and $K_{2}=H \ominus H_{2}$ have infinite dimension.

By Theorem 2.2 there is a unitary $U_{0}: H_{1} \rightarrow H_{2}$ such that $V_{2}=U_{0} V_{1}$ and $\iota_{H_{2}} U_{0}-\iota_{H_{1}}$ is compact.

Moreover, since the subspaces $H_{1}$ and $H_{2}$ satisfy condition 3) in Theorem 2.2. $P_{1}-P_{2}$ is compact and $\operatorname{dim}\left(H_{1} \cap K_{2}\right)=\operatorname{dim}\left(K_{1} \cap H_{2}\right)$. But, as $P_{1}-P_{2}=$ $\left(\mathbf{1}_{H}-P_{1}\right)-\left(\mathbf{1}_{H}-P_{2}\right)$, the subspaces $K_{1}$ and $K_{2}$ themselves satisfy the same condition. Therefore from Theorem 2.2, $K_{1}$ and $K_{2}$ are also ranges of isometries with compact difference or, equivalently, there is a unitary $U_{1}: K_{1} \rightarrow K_{2}$ such that $\iota_{K_{2}} U_{1}-\iota_{K_{1}}$ is compact. But then $U=U_{0} \oplus U_{1}$ satisfies $V_{2} U=V_{1}$ and $U-\mathbf{1}_{H}$ is compact, which proves the direct implication. The converse is trivial.

A dilation argument can be used to prove the following analogous result for contractive perturbations.

Theorem 2.4. Let $V$ be an isometry and $T$ a contraction in $B(L, H)$. Then $T-V$ is compact if and only if there is a contraction $Y \in B(H)$ such that $Y-\mathbf{1}_{H}$ is compact and $T=Y V$. 
Proof. It is enough to prove the direct implication. The equality

$$
\mathbf{1}_{L}-T^{*} T=\frac{1}{2}\left[\left(V^{*}-T^{*}\right)(V+T)+\left(V^{*}+T^{*}\right)(V-T)\right]
$$

shows that $\mathbf{1}_{L}-T^{*} T$ is compact; therefore $D_{T}=\left(\mathbf{1}_{L}-T^{*} T\right)^{1 / 2}$ is compact.

We consider the isometries $V_{1}$ and $V_{2}$ from $H$ to $H \oplus L$ defined by $V_{1}:=\left(\begin{array}{l}V \\ 0\end{array}\right)$ and $V_{2}:=\left(\begin{array}{c}T \\ D_{T}\end{array}\right)$. Then $V_{1}-V_{2}$ is compact, so by Theorem 2.3 there is a unitary $U$ in $B(H \oplus L), U=\left(\begin{array}{ll}U_{11} & U_{12} \\ U_{21} & U_{22}\end{array}\right)$ such that $V_{2}=U V_{1}$, so $T=U_{11} V$.

Obviously $U_{11}$ is a contraction in $B(H)$, and since $U-\mathbf{1}_{H \oplus H}$ is compact, it follows that $U_{11}-\mathbf{1}_{H}$ is compact. Therefore we can choose $Y=U_{11}$, which satisfies all the requirements needed, and the proof is complete.

As a consequence we derive a generalization of the parametric form (1.1) for general compact perturbations.

Corollary 2.5. Let $V$ be an isometry and $K$ a compact operator in $B(L, H)$. Then $V+K$ is an isometry if and only if there is an orthonormal sequence of vectors $\left(e_{n}\right)_{n} \subset H$ and $\left(\alpha_{n}\right)_{n} \subset \mathbb{T}$ such that $\lim _{n \rightarrow \infty} \alpha_{n}=1$ and

$$
K=\sum_{n=0}^{\infty}\left(\alpha_{n}-1\right) e_{n} \otimes V^{*} e_{n} .
$$

Proof. By Theorem 2.3, $V^{\prime}=V+K$ is an isometry if and only if there is a unitary $U$ such that $V^{\prime}=U V$ and $U-\mathbf{1}_{H}$ is compact. It follows that $U-\mathbf{1}_{H}$ is diagonalisable, so there is an orthonormal base $\left(e_{n}\right)_{n \geq 0}$ of $H$ and a sequence $\left(\beta_{n}\right)_{n \geq 0}$ of scalars (the eigenvalues of $U-\mathbf{1}_{H}$ ) such that $U-\mathbf{1}_{H}=\sum_{n=0}^{\infty} \beta_{n} e_{n} \otimes e_{n}$.

It follows that $K=\sum_{n=0}^{\infty}\left(\alpha_{n}-1\right) e_{n} \otimes V^{*} e_{n}$, with $\alpha_{n}=\beta_{n}+1 \in \mathbb{T}$ as eigenvalues for the unitary $U$.

It is obvious that, in the parametric description in Corollary 2.5 for compact perturbations, the finite rank case corresponds to a finite sum in (2.1). This and Corollary 2.5 give one consequence concerning compact perturbations of isometries as iterated rank-one perturbations.

Corollary 2.6. a) Suppose that the isometry $V^{\prime} \in B(L, H)$ is a compact perturbation of the isometry $V \in B(L, H)$. Then $V^{\prime}$ is the norm limit of a sequence $V_{n} \in B(L, H)$ of isometries such that $V_{0}=V$ and $V_{n}$ either coincides or is a rank-one perturbation of $V_{n-1}$ for every $n \geq 1$.

b) In particular if $V^{\prime}$ is a finite rank perturbation of $V$ there is a finite sequence $V_{0}=V, V_{1}, \ldots, V_{n}=V^{\prime}$ of isometries such that each $V_{k}$ is a rank one perturbation of $V_{k-1}$.

\section{ACKnowledgments}

The authors wish to thank G. Cassier for helpful discussions on the subject and the referees for many valuable suggestions that considerably improved the presentation of the paper. 


\section{REFERENCES}

[BT97] Chafiq Benhida and Dan Timotin, Functional models and finite-dimensional perturbations of the shift, Integral Equations Operator Theory 29 (1997), no. 2, 187-196. MR.1472098 (98d:47032)

[BT00] , Finite rank perturbations of contractions, Integral Equations Operator Theory 36 (2000), no. 3, 253-268. MR1753419 (2001f:47011)

[Cla72] Douglas N. Clark, One dimensional perturbations of restricted shifts, J. Analyse Math. 25 (1972), 169-191. MR0301534 (46:692)

[CT04] Gilles Cassier and Dan Timotin, Power boundedness and similarity to contractions for some perturbations of isometries, Journal of Mathematical Analysis and Applications 293 (2004), 160-180. MR2052538 (2004m:47044)

[Nak86] Yoshihiro Nakamura, One-dimensional perturbations of isometries, Integral Equations Operator Theory 9 (1986), no. 2, 286-294. MR0837520 (87k:47031)

[Nak93] _ One-dimensional perturbations of the shift, Integral Equations Operator Theory 17 (1993), no. 3, 373-403. MR1237960 (94h:47019)

Université Bordeaux 1, LAPS, Equipe Signal et Image, 351 cours de la Libération, 33405 Talence cedex, France

E-mail address: Ioana.Serban@laps.u-bordeaux1.fr

Université Bordeaux 1, LAPS, Equipe Signal et Image, 351 cours de la Libération, 33405 Talence cedex, France

E-mail address: Flavius.Turcu@laps.u-bordeaux1.fr 